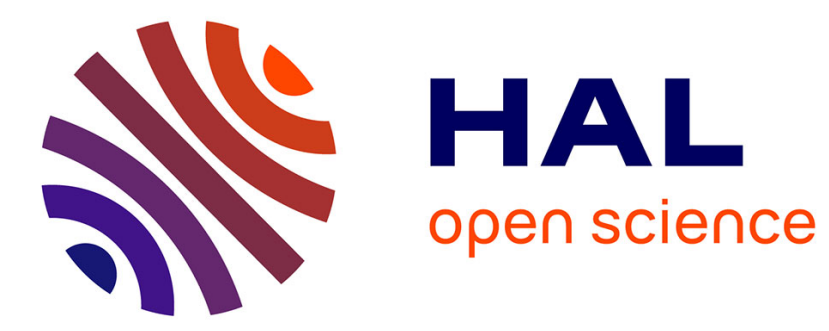

\title{
On Design and Deployment of Fuzzy-Based Metric for Routing in Low-Power and Lossy Networks
} Patrick Olivier Kamgueu, Emmanuel Nataf, Thomas Djotio Ndié

\section{To cite this version:}

Patrick Olivier Kamgueu, Emmanuel Nataf, Thomas Djotio Ndié. On Design and Deployment of Fuzzy-Based Metric for Routing in Low-Power and Lossy Networks. IEEE SenseApp 2015, Oct 2015, Clearwater Beach, Floride, United States. hal-01203409

\section{HAL Id: hal-01203409 \\ https://hal.science/hal-01203409}

Submitted on 23 Sep 2015

HAL is a multi-disciplinary open access archive for the deposit and dissemination of scientific research documents, whether they are published or not. The documents may come from teaching and research institutions in France or abroad, or from public or private research centers.
L'archive ouverte pluridisciplinaire HAL, est destinée au dépôt et à la diffusion de documents scientifiques de niveau recherche, publiés ou non, émanant des établissements d'enseignement et de recherche français ou étrangers, des laboratoires publics ou privés. 


\section{On Design and Deployment of Fuzzy-Based Metric for Routing in Low-Power and Lossy Networks}

\author{
Patrick Olivier Kamgueu \\ LIRIMA MASECNESS Team \\ Lorraine University \\ 615 Rue du jardin botanique, \\ 5600 Villers-les-Nancy, France \\ patrick-olivier.kamgueu@inria.fr
}

\author{
Emmanuel Nataf \\ INRIA MADYNES Team \\ Lorraine University \\ 615 Rue du jardin botanique, \\ 5600 Villers-les-Nancy, France \\ emmanuel.nataf@loria.fr
}

\author{
Thomas Ndie Djotio \\ LIRIMA MASECNESS Team \\ University of Yaounde 1 \\ National Advanced School of Engineering \\ BP 8390 Yaounde, Cameroon \\ tdjotio@gmail.com
}

\begin{abstract}
Minimizing the energy consumption and hence extends the network lifetime is a key requirement when designing an efficient sensor network protocol. QoS-aware routing in Wireless Sensor Network (WSN), aims to take into account other networks performance aspects as minimizing end-to-end delay (as well as jitter), reducing packet loss rate while minimizing the energy consumption of the network during data transmission. These objectives are sometimes conflicting, and therefore tradeoffs must be made between energy conservation and QoS considerations. The general problem can be reformulated as a Multi-Constrained Optimal Path problem (MCOP), and is known as NP-complete. The latter raises a real challenge, as sensor nodes are very limited in resources capabilities, we propose to use fuzzy inference mechanism to seek a good tradeoff between all given metrics and constraints. This paper discusses the implementation of combining several routing metric, using fuzzy logic to design a RPL objective function, the routing standard for the Internet of Things. The proposal is integrated on Contiki operating system and his deployment were performed on a real world indoor WSN. Obtained results show improvements compared to the common implementation of the RPL protocol, and demonstrate relevance of our contribution.
\end{abstract}

\section{INTRODUCTION}

These recent years, Wireless Sensor Networks (WSN) became an attracting field that arouse interest of the scientific and industrial communities. Topology of such network is a collection of large number of sensor nodes deployed in the target area to detect some physical phenomenon. Usually, a particular node called the sink attached to a base station, receives all network data through neighbor nodes using multihop radio communication. Nodes are generally small devices with low-cost, low-power that are equipped with only a limited data processing capabilities and low transmission rate, on a battery operated energy supply and scarce memory. The introduction of IP-based protocols and open standards led to the adoption of IPv6 over Low power Wireless Personal Area Networks (6LoWPAN) and IPv6 Routing protocol for Low power and lossy networks namely RPL (pronounce ripple) [20], standardized by Internet Engineering Task Force (IETF). This opens new opportunities in various fields and application areas, such as home automation, smart cities and power grids, healthcare, critical areas control (nuclear power plan, forest fires, disaster prevention, etc.).

Routing protocol is a key issues for WSN, and RPL [20] was designed to take into account the unique characteristics of this kind of network. A number of metrics [19] are intended to be accounted by the protocol during the network topology building phase. These metrics are implemented as an objective function $(\mathrm{OF})$ with the purpose to setting up various performance objectives for the network, in order to satisfy the requirements of the target application. So far, only two objective functions are specified and standardized for RPL: The first uses hop-count as routing metric also called of 0 [18], and the other uses the expected number of transmission needed to successfully send a packet to its destination (ETX) namely Minimum Rank with Hysteresis Objective Function [6]. The use of other defined criteria (energy, latency, throughput, etc.) are left to implementer, also the possibility of combining several metrics into one, to ensure quality of service (QoS) or meet application requirements, is not well-addressed.

Several traffic flows with different QoS requirements may share the same WSN, including for example, both periodic and non-periodic data. For instance, one application can report periodically the temperature and lighting, while reacting to sudden events like threshold crossing or the entering of a person in a room. The set of services to be fulfilled when transmitting a stream of packets from source to destination are diverse, such as reduce end-to-end delay and maintain jitter steady, minimize packet loss, while extending the network lifetime. But these requirements are sometimes conflicting issues under a WSN context. For instance, one can improve reliability by increasing the number of maximum allowable retransmission or using higher transmission power levels. However, in both cases, more energy will be expended, thus reducing the overall network lifetime. So, tradeoffs must be made between energy conservation and other QoS considerations. The problem becomes: how to make these tradeoffs at runtime, or find integrated performance metrics that accounts the above requirements online. Traditional wired and wireless network QoS algorithms and techniques are not directly applicable on WSN due to their unique features and resource constraints.

Combining several routing metrics in RPL to improve QoS has already been investigated in the literature [11], and falls into two forms of combination: additive and lexicographic. In this paper we propose to take advantage of fuzzy logic to solve it and contribute to improve RPL by considering multiple objectives. This approach is motivated in that, using this paradigm, with a small memory footprint, we can seek for a halfway between several criteria, even antagonistic (this is 
not the case with the aforementioned combination methods). A necessary condition with the additive composition is that basic metrics follow the same dimming direction (growing or decreasing). Lexicographic composition overcomes the latter restriction, but the main shortcoming with this approach is that, in most cases when comparing two composite metrics (wrote as a vector), only the first metric component is considered, subsequent values are used to break the tie.

The remainder of the paper is organized as follows. Section 2 presents related works on combining metrics for routing in WSN, as well as works that use fuzzy inference system for routing design. Section 3 describes an overview of RPL standard. In section 4 , we describe the proposed objective function design, followed by implementation parameters, experiment results and discussions in section 5. Finally, we conclude and discusses future directions in section 6 .

\section{RELATED WORK}

In order to account QoS in RPL, Karkazis et al. [11] propose to use additive and lexicographic composition to optimize more than one performance aspects. They also indicate conditions that basic metrics must hold to satisfy properties of convergence, optimality and loop freeness. In the additive approach, the composite metric $M$ is written as a linear combination of basic metrics $\left(M=\sum_{i} \alpha_{i} m_{i}\right.$, where $m_{i}$ are basic metrics, each weighted by given coefficients $\alpha_{i}$ ). The main shortcoming of this scheme is that, basic metrics must necessarily be defined on the same order relation, thus it restricts the type of metrics to be considered. In the lexicographic approach, given two basic metrics $M=\left\langle m_{1}, m_{2}, \ldots, m_{k}\right\rangle$ and $N=\left\langle n_{1}, n_{2}, \ldots, n_{k}\right\rangle$ with the respective order relation $\preceq_{1}, \preceq_{2}, \ldots, \preceq_{k}$, metric elements are evaluated sequentially: $M \preceq_{\text {lex }} N \Leftrightarrow\left(m_{1} \preceq_{1} n_{1}\right.$ or $\left.m_{1}=n_{1} \wedge m_{2} \preceq_{2} n_{2}, \ldots\right)$. So, the subsequent metrics values are accounted only to break the tie. Hence, most of the time many metrics are not considered.

In that context, Link Estimation and Parent Selection protocol [22] combines hop-count and Link Quality Indicator (LQI) in lexicographic manner to select the best next hop. Firstly, the source node selects neighbouring with the minimum hop towards the sink node as its parent, if there is more nodes having the same hop-count value, it's the one with the largest LQI is chosen. The main disadvantage of this protocol is the early death of nodes and the unbalanced energy dissipation. RPLRE [21] overcomes the latter and suggests to use a probability selection scheme that take into account the residual energy when choosing next hop, besides LQI and hopcount. The probability scheme improves selection process and avoids to choose the same node most often. The result is a more balanced energy consumption among potential parents, and the network lifetime is thus extended.

Aslam et al. [2] propose a composite metric that uses multiple parameters or QoS constraints to find an optimal route for the Optimized Link State Routing (OLSR) protocol. OLSR protocol is natively based on hop-count, authors computed the composite value as a linear combination of maximum available bandwidth, minimum delay and jitter. Contrary to the previous methods, EARQ [9] a novel routing protocol for wireless industrial sensor networks, consider basic metrics criteria separately, instead as a unique combined metric value.
Protocol aims to provide real-time, reliable delivery of a packet while considering energy awareness. Firstly, the path with a lower energy cost is selected according to a probabilistic model, in addition only paths that may deliver packets in time are selected to achieve real-time requirement. Moreover, source node may send a redundant packet via alternate path if the reliability is not meet. EARQ supposes that every node knows it location and rely on a GPS mechanism or a location process for that, but this assumption is not always feasible for many WSN deployments.

There are many growing interest for the integration of artificial intelligence techniques or control process like fuzzy rule-based systems to design protocols for WSN. So, GAFO [5] uses a genetic adaptive fuzzy hop selection scheme, to make optimal choices for robust packet transmission in WSN involved in varying channel conditions. Authors describe a fuzzy system engine that takes signal to noise ratio and outage probability as input, to determine the possibility of a neighbor node to be selected as the next hop for data forwarding. Experiments show that in the same conditions, this protocol outperforms the crisp approach on average by $20 \%$ for reliability and $15 \%$ for total energy consumption. Likewise, a cluster head election method using fuzzy logic has been introduced by Gupta et al. [7] to overcome the defect of LEACH [8], a popular cluster head selection technique. The main idea for the LEACH protocol is that nodes are elected depending on a stochastic model and use localized clustering. The consequence is that some cluster heads may be very close to each other or may be located in the edge of the WSN. This careless cluster heads distribution could not maximize energy efficiency. Other fuzzy-based schemes [3], [17] were proposed to improve election process involved on LEACH. Those have proved that the network lifetime can be efficiently extended using fuzzy variables (concentration, energy and node centrality). Unfortunately, LEACH is not applicable to networks deployed in large regions, since it uses single-hop routing where node can transmit data directly to cluster head, afterwards the latter transmit data to the sink.

Contrary to LEACH were each cluster head must directly sends data to sink, EDARP [23] establishes a Fuzzy Spanning Tree that uses the energy and distance to construct a routing path over all cluster heads. These two criteria are used to generate a fuzzy election number and lead to the selection of the best parent into the routing tree. Hence, energy consumption is balanced among all nodes by keeping rotation in cluster head election and parent's node selection. FEAR [1] proceeds slightly differently. Rather than using a clustering mechanism to build the hierarchical topology, FEAR protocol directly builds a logical tree topology among all network nodes. A ranking-based system that relies on fuzzy inference is used, so that nodes rank their neighbors by considering both neighbors depth and power consumption. This fuzzy ranking system is used to construct and maintain the tree topology. Compared to RPL, FEAR generates more control messages which implies a greater power consumption. In addition, the protocol uses a node identification (ID) construction model where a node's ID is computed based on the node's parent ID. Unfortunately, when a parent node dies, all nodes in its subtree must recomputed their ID as soon as a new parent is found, involving more processing and communication overhead. 


\section{RPL OVERVIEW}

RPL [20] is a distance vector routing protocol optimized for low power and lossy network, where multipoint-to-point is the dominant traffic pattern. The protocol also support pointto-multipoint traffic pattern using destination advertisement mechanism, and provide a basic structure for point-to-point route. The network topology is organized as one or more Destination Oriented Direct Acyclic Graph (DODAG) each rooted at a single point, that acts as sink for the topology. Three new types of ICMPv6 messages are defined and manipulated:

- DODAG Information Object (DIO) used to create and maintain upward routes.

- DODAG Destination Advertisement Object (DAO) used to install downward routes.

- DODAG Information Solicitation (DIS) actively used by a node wishing to join the network or asking for up to date informations.

The topology building starts at the root (initially, the only router which is part of a DODAG), that sends DIO messages in its neighbourhood. This message conveys all common configuration parameters, including root ID, mode of operation, timers values, etc. Upon receipt of a number of such messages, neighbour nodes may participate in the DODAG according to the objective function $(\mathrm{OF})$, select theirs parents and then start to issue their own DIO messages. This process spreads gradually to cover the whole network while new nodes join the DODAG. Only one node among parent's nodes (the preferred parent) acts as the next-hop on the path towards the root.

RPL pro-actively creates and maintains the topology, by regularly sending ICMP control messages in the vicinity. The spreading rate is governed by the trickle algorithm [12], that reduce the overhead induced by control messages. This is done by sending DIO less often when the topology is steady, but reacts and quickly spreads informations on topology change or when inconsistencies are detected.

An important point is when a given node receives more than one consistent DIO, each from a different neighbour and must choose which one would be the preferred parent. This choice is governed by the objective function that specifies how this node selects its best parent into the parent set, and calculates its own rank (a gradient representing its relative position with respect to the root) from the parent's rank. Different criteria also called routing metrics are defined [19] to capture link or node characteristics on the path for parent selection purpose. The rank computation is derived from the set of these selected metrics, and must monotonically decrease as we move toward the root. This last property enables the routing structure to maintain its acyclic nature, thus helps to avoid routing loops.

Unlike existing OFs [6], [18] that rely on a unique routing metric to construct the DODAG, so, we want to combine several metrics into RPL by taking into account more than one performance aspect. The IETF ROLL working group has left open to the implementer the definition of new OFs, only the acyclic nature of the graph must be preserved. Routing metrics and constraints have been proposed by the working group, but the possibility to combine them is also not welladdressed. The next section shows how we design a such $\mathrm{OF}$ using fuzzy inference system.

\section{Routing Metric Design}

\section{A. Fuzzy Inference System}

Fuzzy logic reasoning allows us to transform several input variables (delay, ETX and energy) into one (Quality). The fuzzy inference system consists on several steps.

- Fuzzification: take a crisp value input and determine its degree of membership (fuzziness) for the appropriate fuzzy sets.

- Fuzzy inference: Apply combination rules to "fuzzified" inputs and compute a fuzzy output.

- Aggregation: If an output depends on more than one rule, this step unifies all values into one.

- Defuzzification: Convert the fuzzy output obtained at the previous step into a crisp value.

In this paper, due to its simplicity and efficiency, we use the most common fuzzy inference method, namely Mamdani model [13].

\section{B. Composite metric design}

To illustrate fuzzy inference reasoning, we consider the network topology depicted in the figure 1 . Node $\mathrm{N}$, in order to send data to the final destination $\mathrm{S}$, must select the next hop (either P1 or P2) as preferred parent. This choice is governed by received informations as shown. We would like to know which parent is the most suitable next hop based on those criteria (energy, delay and ETX), according to the fuzzy inference engine.

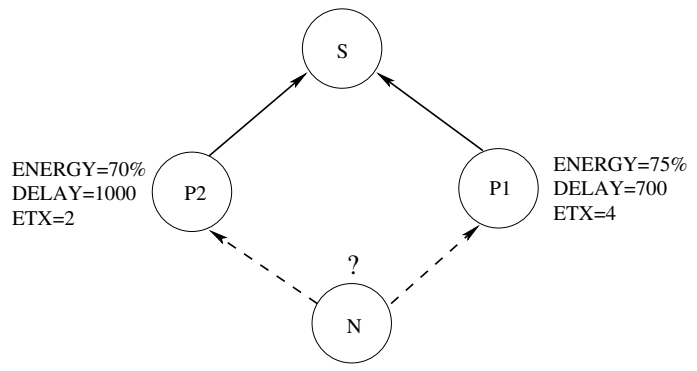

Fig. 1: Parent Selection Process

1) Linguistic variables: Node's performances knowledge are represented as linguistic variables. In this case, we consider the following three metrics (or linguistic variables in the fuzzy inference vocabulary):

- $\quad$ ETX - The expected number of required transmissions before a packet reaches the destination. It assesses the transmission accuracy.

- Delay - The average time for a packet to reach its destination. 
- Energy - The energy cost of the path, also energy of the node having the smallest remaining battery level on the path.

We use cross-layer mechanisms to retrieve ETX and delay from data link and network layers. To estimate ETX, a sending node record number of transmission before receiving an acknowledgement. The average value is computed as an EWMA (Exponentially Weighted Moving Average) over the time. The one-hop delay is computed as the required time to send a packet and received the acknowledgement. This time includes MAC contention mechanisms. The overall end-to-end delay is the delay up to the sink.

Node's energy is estimated based on the online energy estimation model [16] and its implementation we conducted in [14], [10]. The model takes into account the energy spent under a constant current load at each node state (transmitting, receiving, idle and sleeping). We also consider the recovery effect which assesses the energy recovered during inactivity period.

2) Fuzzification process: To avoid the complexity of directly combine the selected three linguistic variables into one, we perform the fuzzification process in two stages, as shown in the figure 2.

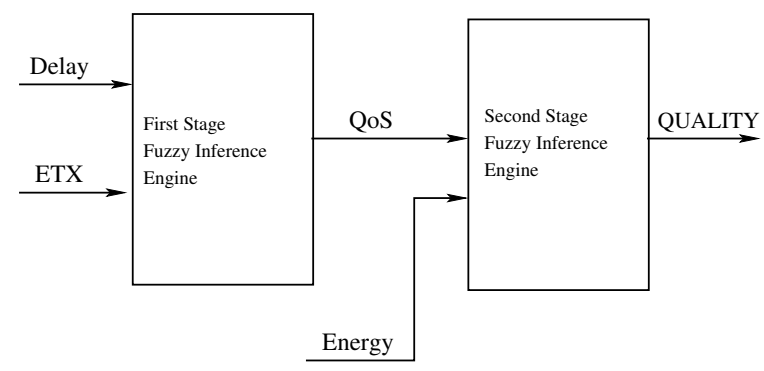

Fig. 2: Fuzzy Inference Engine

First stage of fuzzification: On the first stage, we combine delay and ETX as inputs to compute QoS, which is taken in its turn as input for the next stage. The linguistic variables used to represent delay are divided into short, average and long membership functions, and ETX fall into small, average and high. Figure 3 depicts their membership functions normalized by the number of hops $(h c)$ upwards to the sink, since delay (respectively ETX) is computed at the path level. Table I illustrates the relationship between these two linguistic variables for the computation of QoS. This table is built according to the desired rules, easy to express, and which depend on the knowledge of an expert. For instance, a long delay and high ETX implies a very slow QoS. So, shorter is the ETX and smaller is the delay, better is the QoS to consider.

\begin{tabular}{|c||c|c|c|}
\hline ETX / Delay & short & average & long \\
\hline \hline small & very_fast & fast & average \\
\hline average & fast & average & slow \\
\hline high & average & slow & very_slow \\
\hline
\end{tabular}

TABLE I: QoS Output Metric

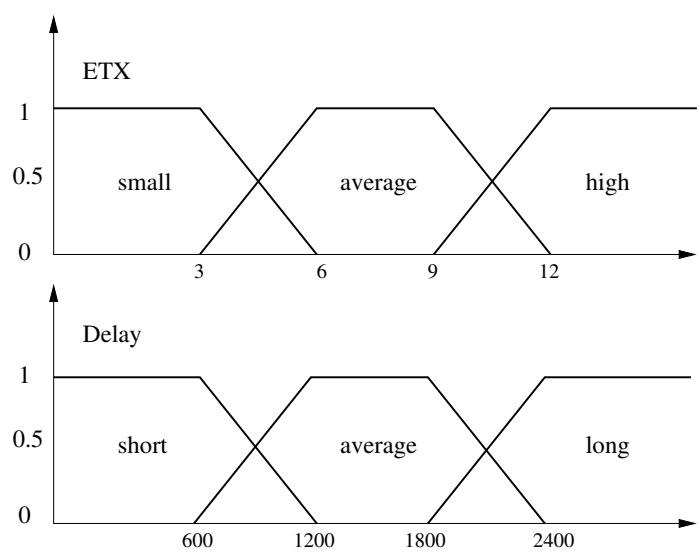

Fig. 3: One-hop membership functions

For instance, considering a crisp value ETX, formula 1 indicates how is its the level of membership in the average fuzzy set, at one hop (ie. $h c=1)$. Similar formulas establish the level of membership for others ETX fuzzy sets (small and high), as well as delay and energy linguistic variables.

$$
\text { average }(\text { et } x)= \begin{cases}0 & \text { if } \text { et } \leq 3 \\ \frac{e t x-3}{6-3} & \text { if } 3<\text { et }<6 \\ 1 \frac{\text { if } x-12}{9-12} & \text { if } 9 \leq \text { et } x \leq 9 \\ 0 & \text { if } \text { et } x \geq 12\end{cases}
$$

For the example provided in figure 1, node $\mathrm{N}$ computes as level of membership small (etx) $=0.66$, average $(e t x)=0.33$, and high $(e t x)=0$ for the parent node P1. The same types of computations for P1's delay allow us to determine as respective fuzzy sets short, average and long, the values $0.83,0.16$ and 0 .

Since QoS relates to ETX and delay, the previously computed membership functions are combined according to the table I. The Mamdani model allows us to use the minimum operator as the composition function, and maximum for the aggregation operator. For instance, formula 2 indicates how to compute average $(Q \circ S)$ fuzzy set from inputs. In this case, three rules are fired, we aggregated them with the maximum operator. Likewise, we establish formulas for $Q o S$ fuzzy sets ranging from very_fast to very_slow.

$$
\operatorname{average}(Q o S)=\max \left(\begin{array}{c}
\min (\text { high }(\text { etx }), \text { short }(\text { delay }) \\
\min (\operatorname{avg}(\text { etx }), \text { avg }(\text { delay })) \\
\min (\operatorname{small}(\text { etx }, \text { long }(\text { delay }))
\end{array}\right)
$$

For our illustrative topology in figure 1, node N computes three non-zero QoS membership functions concerning neighbour P1: very_fast $(Q \circ S)=0.66$, fast $(Q \circ S)=0.33$, and average $(Q \circ S)=0.16$. These values are defuzzified (as described in $\S I V-B 3)$ into a single QoS output $(Q \circ S=0.78)$, and then used in the next fuzzification stage.

Second stage of fuzzification: As the second stage of the fuzzy inference system, we combine the previously computed QoS with the energy linguistic variable to provide QUALITY. For a given node, energy could be low, medium or full, 
and the output values for QUALITY is divided into seven levels ranging from awful to excellent. Table II shows how to derive QUALITY based on QoS and energy.

\begin{tabular}{|c||c|c|c|}
\hline QoS / Energy & low & medium & full \\
\hline \hline very_slow & awful & bad & average \\
\hline slow & bad & degraded & average \\
\hline average & degraded & average & acceptable \\
\hline fast & average & acceptable & good \\
\hline very_fast & average & good & excellent \\
\hline
\end{tabular}

TABLE II: QUALITY Output Metric

3) Defuzzification process: All fuzzy values obtained after aggregation step are converted into a single crisp output. The most common and accurate defuzzication method uses the centroid, where the result is the center of gravity of the polygon drawn using fuzzy values of the output membership function. Figure 4 illustrates the defuzzification process for QUALITY linguistic variable and formula 3 how to compute the final crisp value $Q$. Output values range from 0 to 100 and indicate how is the level of quality to choose a neighbour as the next hop, according to the selected metrics.

$$
Q=\frac{\sum_{i=1}^{k} \alpha_{i} \times Q U \operatorname{ALITY}\left(\alpha_{i}\right)}{\sum_{i=1}^{k} Q U \operatorname{ALITY}\left(\alpha_{i}\right)}
$$

where $k$ is the number of fired rules, $\alpha_{i}$ is the domain value related to the $i^{t h}$ rule, and $Q U A \operatorname{LITY}\left(\alpha_{i}\right)$ is the level of trustiness value according to this corresponding domain.

For the proposed topology, three membership functions:

acceptable (QUALITY) $=0.25$,

$\operatorname{good}($ QUALITY $)=0.70$

excellent (QUALITY) $=0.30$,

are fired as P1's QUALITY output. The center of gravity for the depicted region is 77. Similar computations produce value 70 for $\mathrm{P} 2$. So, the best next hop for $\mathrm{N}$ according to the fuzzy inference engine on these three criteria (ETX, delay and energy) is $\mathrm{P} 1$. By considering the lexicographic composition of metrics ETX, delay and energy respectively, this would have led to choose P 2 as the next hop.

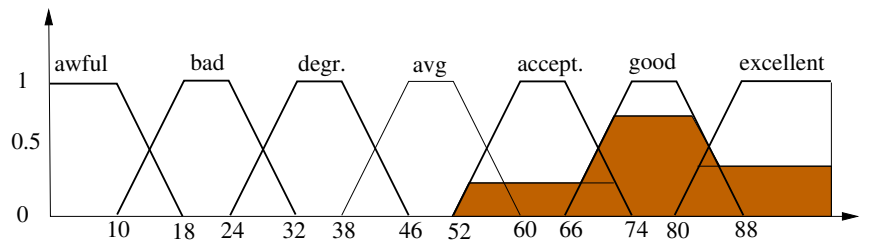

Fig. 4: QUALITY defuzzification

\section{EXPERIMENTS RESULTS}

\section{A. Network setup}

To evaluate the proposed combined metric, we deployed a real indoor WSN of twenty-eight sensor nodes as depicted by figure 5. They are placed in fourteen offices, two nodes per office, the displayed labels are node's ID and links indicate the next hop choose by node at a given time. Nodes are TelosB MTM-CM5000-MSP type, equipped with MSP430 16-bit Texas Instruments micro-controller, CC2420 radio frequency chips, various sensors (temperature, relative humidity and light sensor), one USB interface and operates on two AA Batteries. RF power range between 0 through $-25 \mathrm{dBm}$ (software configurable) and allows emission range from 20 to $30 \mathrm{~m}$ in indoor environment. Sensor nodes, located in fixed place without mobility, sense data and transmit them to the sink for processing every 5 minutes. Sink node is directly attached to the gateway, and thus is assumed to have unconstrained battery power. Nodes run Contiki version 2.7, a well-known operating system for embedded devices [4]. Table III summarizes other parameters of the protocol stack.

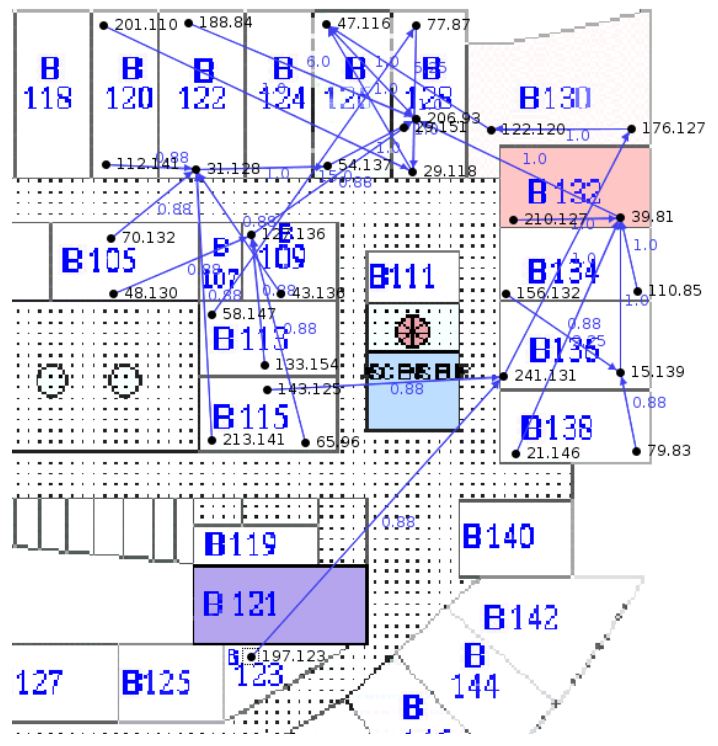

Fig. 5: Node placement

\begin{tabular}{|c||c|}
\hline \multicolumn{1}{|c||}{ Settings } & Values \\
\hline \hline Application Layer & Periodic Sensor Data collection \\
\hline Transport Layer & UDP \\
\hline Network Layer & $\mu \mathrm{IPv6}+$ 6LoWPAN + RPL (routing) \\
\hline MAC Layer & non-persistent CSMA \\
\hline Radio Duty Cycle & ContikiMAC \\
\hline PHY + Radio chip & IEEE 802.15.4 w/ CC2420 \\
\hline
\end{tabular}

TABLE III: Protocol stack

\section{B. Performance evaluation}

We develop two scenarios of a data collection application, were each node sense its vicinity and transmit measured environmental parameters data (temperature, brightness and humidity) to the sink through neighbors using multihop links, during slightly more than two weeks. In the first scenario, network is organized according to the standard RPL that uses ETX as the single routing metric. The second scenario instead uses the proposed combined metric scheme: ETX, Delay and Energy according to the Fuzzy Inference Engine. We are interested to evaluated the reliability of the application to collect sent data, the stability of the routing by assessing the 
number of best parent change over the time and finally the network lifetime by looking at energy depletion of nodes.

1) Packet Loss Ratio: As depicted by figure 6, experiment results indicate that the combined metric scenario obtain better results in packet loss ratio than the native ETX RPL. While the former stabilizes the loss rate around $5 \%$, in the latter this loss is almost three times higher. When looking at the network startup phase, we can notice that the proposed scenario behaves better. Indeed, the combined metric based RPL remains relatively stable, whilst during the three first days, there is a high loss ratio for the ETX based routing (about 25\%). This behavior of the ETX-based routing during the first three days is due to the bad routes selected and shows its slowness to reach to a steady state.

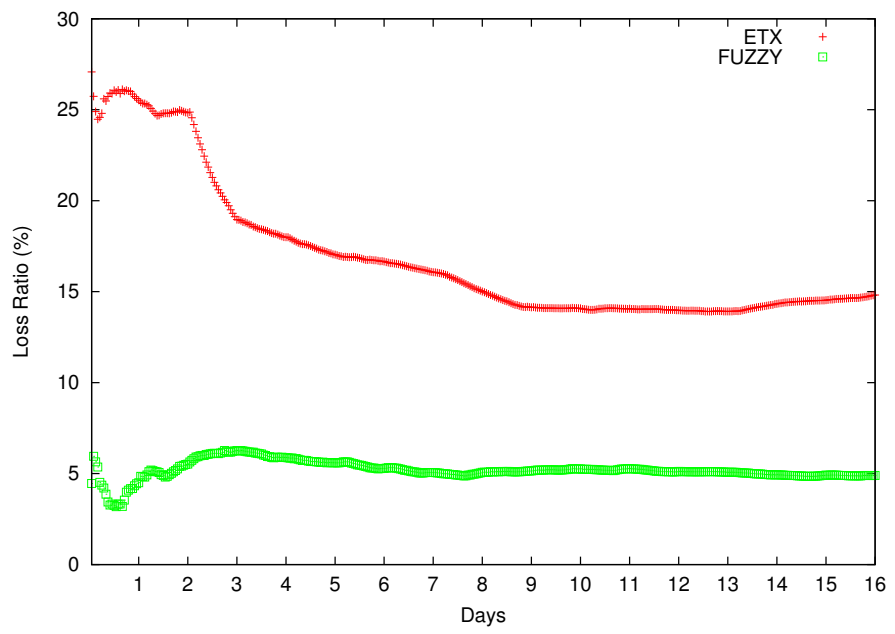

Fig. 6: Packet loss ratio over time at sink node

2) Routing Stability: This performance metric allows us to assess the number of best parent changes in the network over the time. A high variation rate reveals an unstable topology and is not desirable, since it can impact the packet delivery and routing table flapping. As shown in figure 7, the number

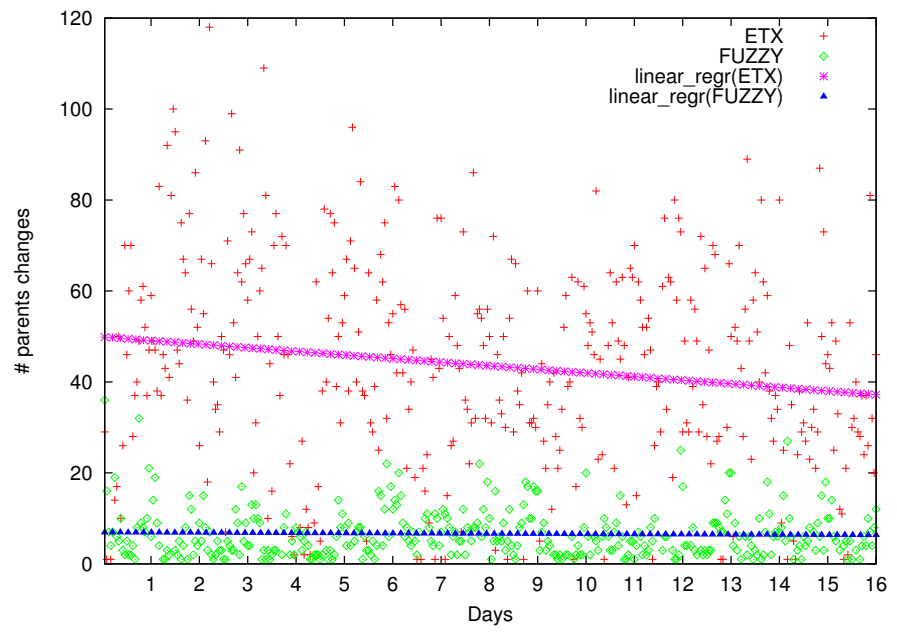

Fig. 7: Number of parent changes per hour of best parent changes remains relatively low and rather steady over time for the fuzzy-based scenario, compared to the ETXbased scenario. The picture also displays the linear regression of number of parent changes distribution. We can see that, the number of parent changes per hour is more higher, with an average of 43.52 for the native ETX-based metric, whereas the same average is 6.63 for the combined metric experiment.

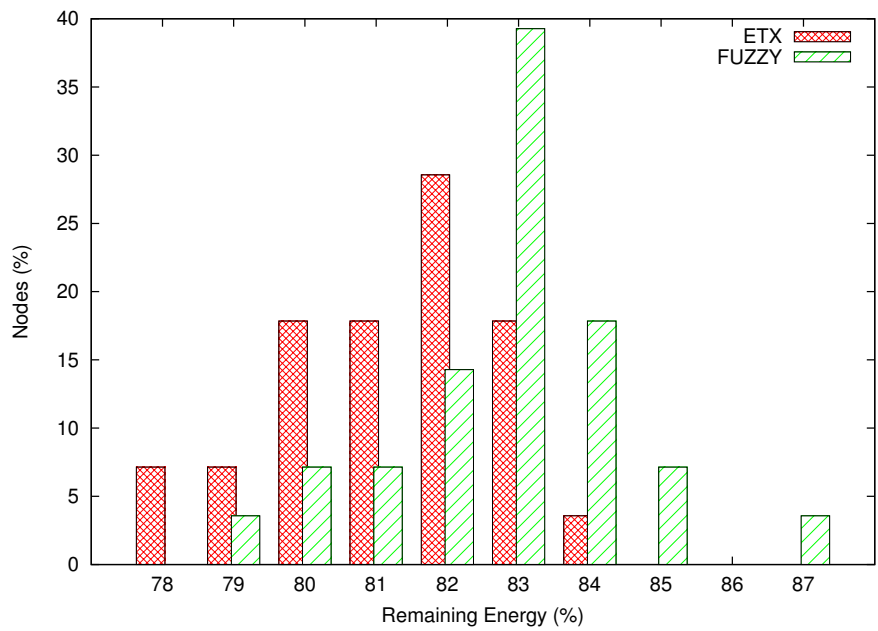

Fig. 8: Remaining power distribution

3) Average Remaining Power: All nodes start with the same power level (100\% battery power level). Figure 8 depicts the remaining power distribution of nodes after sixteen days of experiments. It is clear that, the pyramid induced by the drawn histogram is more to the left side for the fuzzy-based metric than the ETX scenario. Thus, the proposed combined metric is more energy conservative than the native ETX metric. More specifically, in the combined metric scenario, $67.85 \%$ of nodes have their energy higher than $83 \%$ of the initial battery level, while this proportion is only $21.42 \%$ for the ETX-based routing. Moreover, the combined metric scenario maintains a great number of nodes $(39.27 \%)$ at a same high power level $(83 \%)$. Likewise, we can denote that weak power nodes (having their remaining battery power level $\leq 81 \%$ of the initial power level) in the ETX based scenario (50\% nodes) outnumber the combined metric scenario ( $17.85 \%$ nodes). This shows that the proposed combined metric is more power efficient than the native ETX based routing, since it accounts battery level in addition to other criteria.

4) End-to-end delay: Delay is another metric that we consider when selecting the best next hop according the three selected parameters (in addition to energy and ETX). The real challenge to evaluate end-to-end delay in the real WSN deployment lies in the fact that all nodes must be synchronized or share the same reference clock. This requires the implementation of additional mechanisms that would be very expensive in terms of memory and processing resources, which already are very limited on the deployed sensor nodes. For those reasons and for simplicity purposes, we emulated the deployed WSN on similar conditions using Cooja [15], a popular WSN simulator environment. Nodes share the same image code as the live deployment, however they also share the same clock with Cooja, which enables us to accurately 
evaluated the end-to-end delay. Figure 9 shows the cumulative discrete function (CDF) of end-to-end delay. Although the gap between the two developed scenarios is not very large, the proposed fuzzy-combined metric behaves better. For instance, we can see that in the fuzzy-based metric, $75 \%$ of packet have a delivery times less or equal to $1 \mathrm{~s}$, where this proportion is $68 \%$ for the ETX-based metric. Note that, since fuzzy-based scenario have a better delivery ratio as shown in $\S \mathrm{V}-\mathrm{B} 1$, the computed end-to-end delay in this scheme is more accurate than ETX-based ones, because more packets are accounted.

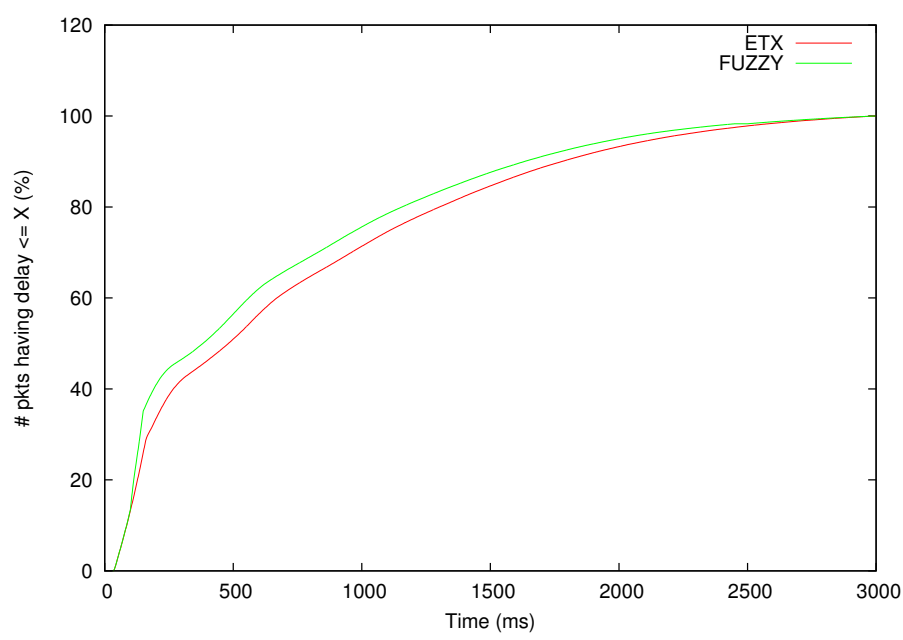

Fig. 9: CDF of end-to-end delay

\section{CONCLUSION}

This paper describes the design and implementation of a new RPL objective function that combines several metrics to optimize more than one network performance aspects. The proposed solution uses fuzzy inference system to merge expected transmission count, delay and node's remaining power into one unique value. We performed a real sensor network deployment in indoor environment, to assess the metric. Experiment results shows that the proposed combined metric outperformed the ETX based routing on packet loss ratio, routing stability, energy efficiency and even on end-to-end delay. The proposed fuzzy inference system is used to find a tradeoff between input metrics which can be antagonistic. In our future works we aim to find a way to favor some metrics compared to others, and thus better tune the contribution of each metric. Moreover network can generate several traffic flows which don't have the same QoS requirements. For instance, some flows must comply with strict end-to-end delay requirements, while other must meet the transmission accuracy. It would be very interesting to investigate how to account those flows in regard to their requirement and take them into account at runtime.

\section{REFERENCES}

[1] I. M. Almomani and M. K. Saadeh, "Fear: fuzzy-based energy aware routing protocol for wireless sensor networks," Int. Journal of Coтmunications, Network and System Sciences, vol. 4, pp. 403-415, 2011.

[2] N. Aslam, W. Phillips, and W. Robertson, "Composite metric for quality of service routing in olsr," in Proc. of Canadian Conference on Electrical and Computer Engineering, Niagara Falls Ontario, Canada, May 2004, pp. $759-762$.
[3] M. Basirnezhad and M. N. Torshiz, "Energy efficient cluster head election using fuzzy logic in wireless sensor networks," International Journal of Computer Science and Information Security, vol. 9, no. 5, pp. 255-260, may 2011.

[4] A. Dunkels, B. Grönvall, and T. Voigt, "Contiki - a lightweight and flexible operating system for tiny networked sensors." in Proc. of $L C N$, 2004.

[5] D. S. Ghataoura, Y. Yang, and G. Matich, "Gafo: genetic adaptive fuzzy hop selection scheme for wireless sensor network," in Proc. of 5th IEEE International Wireless Communication and Mobile Computing conference (IWCMC), Leipzig, Germany, june 2009.

[6] O. Gnawali and P. Levis, "The Minimum Rank with Hysteresis Objective Function,” RFC 6719, Internet Engineering Task Force, sep 2012.

[7] I. Gupta, D. Riordan, and S. Sampalli, "Cluster-head election using fuzzy logic for wireless sensor networks," in Proc. of 3rd IEEE Annual Communication Networks and Services Research Conference, Halifax, Canada, may 2005.

[8] W. Heinzelman, A. Chandrakasan, and H. Balakrishnan, "Energyefficient communication protocol for wireless microsensor networks," in Proc. of 33rd Hawaii International Conference on System Sciences (HICSS), 2000, pp. 3005-3014.

[9] J. Heo, J. Hong, and Y. Cho, "Earq: Energy aware routing for realtime and reliable communication in wireless industrial sensor networks," IEEE Trans. on Industrial Informatics, vol. 5, no. 1, pp. 3 - 11, Feb. 2009.

[10] P. Kamgueu, E. Nataf, T. Djotio, and O. Festor, "Energy-based metric for the routing protocol in low-power and lossy network," in Proc. of 2nd Sensornets, Barcelona, Spain, Feb. 2013.

[11] P. Karkazis, P. Trakadas, H. C. Leligou, L. Sarakis, I. Papaefstathiou, and T. Zahariadis, "Evaluating routing metric composition approaches for qos differentiation in low power and lossy networks," Trans. of Wireless Networks, vol. 19, no. 6, pp. 1269-1284, August 2013.

[12] P. Levis, T. Clausen, J. Hui, O. Gnawali, and J. Ko, "The Trickle Algorithm," RFC 6206, Internet Engineering Task Force, mar 2011.

[13] E. H. Mamdani, "Application of fuzzy logic to approximate reasoning using linguistic synthesis," IEEE Transaction on Computing, vol. C-26, no. 12, pp. 1182 - 1191, 1977.

[14] E. Nataf and O. Festor, "Online estimation of battery lifetime for wireless sensors network," in Proc. of 2nd Sensornets, Barcelona, Spain, Feb. 2013.

[15] F. Osterlind and A. Dunkels, "Cross-level sensor network simulation with cooja," in Proc. of 31st IEEE Conf. SenseApp, Tampa, Florida, 2006, pp. $641-648$.

[16] J. Rahmé and N. Fourthy, "Energy management for battery-powered embedded systems," in Proc. of IEEE WCNC, Sydney, Apr. 2010, pp. $277-324$

[17] G. Ran, H. Zhang, and S. Gong, "Improving on leach protocol of wireless sensor networks using fuzzy logic," Journal of Information \& Computational Science, vol. 7, no. 3, pp. 767-775, 2010.

[18] P. Thubert, "Objective Function Zero for the Routing Protocol for LowPower and Lossy Networks (RPL)," RFC 6552, Internet Engineering Task Force, mar 2012

[19] J. Vasseur, M. Kim, K. Pister, N. Dejean, and D. Barthel, "Routing Metrics Used for Path Calculation in Low-Power and Lossy Networks," RFC 6551, mar 2012.

[20] T. Winter, P. Thubert, A. Brandt, J. Hui, R. Kelsey, P. Levis, K. Pister, R. Struik, J. Vasseur, and R. Alexander, "RPL: IPv6 Routing Protocol for Low-Power and Lossy Networks," RFC 6550, Internet Engineering Task Force, mar 2012.

[21] C. Yan, J. Hu, L. Shen, and T. Song, "Rplre: A routing protocol based on lqi and residual energy for wireless sensor networks," in Proc. of International Conference on Information Science and Engineering (ICISE), Nanjing, China, Dec. 2009, pp. 2714 - 2717.

[22] T. Yan and L. Sun, "Principle and performance evaluation of routing protocol in tinyos," Trans. on Computer Engineering, vol. 33, no. 1, pp. 112 - 114, Jan. 2007.

[23] M. Zeynali, L. M. Khanli, and A. Mollanejad, "Edarp : novel energy and distance-aware routing protocol in wireless sensor network," in 2 nd ICIS : Information Technology, Culture and Human, IEEE, Ed., Seou, Korea, november 2009. 